\title{
Lo que importa es la pregunta. Aportes de la Economía Política Internacional en Latinoamérica para el debate en el contexto de crisis de la globalización ${ }^{1}$
}

What matters is the question. Contributions of International Political Economy in Latin America, for the debate in the context of the crisis of globalization

\author{
Julieta Zelicovich ${ }^{2}$
}

Resumen: En el artículo se analizan las características del desarrollo reciente de la Economía Política Internacional en la región latinoamericana. Se busca resaltar la contribución de la Economía Política Internacional a las Relaciones Internacionales, para cuestionar los procesos de transformación en curso, definidos como crisis de globalización. Para ello se recopilan las preguntas de investigación de una serie de trabajos de reciente publicación, a través de las cuales puede apreciarse la capacidad crítica de este campo de estudio.

Palabras claves: Economía Política Internacional, Relaciones Internacionales, Crisis de globalización.

\begin{abstract}
The article analyzes the characteristics of the recent development of the International Political Economy in the Latin American region. It seeks to highlight the contribution made by International Political Economy to International Relations, to question the ongoing processes of transformation, which are defined as a crisis of globalization. To this end, several research questions from a series of recently published works are collected, through which the critical capacity of this field of study can be appreciated.
\end{abstract}

Key words: International Political Economy, International Relations, Crisis of globalization.

\footnotetext{
${ }^{1}$ Recibido: $17 / 08 / 17$. Aceptado: 23/11/17

${ }^{2}$ Doctora en Relaciones Internacionales, Universidad Nacional de Rosario. Magíster en Relaciones Comerciales Internacionales, Universidad Nacional de Tres de Febrero. Investigadora Asistente en CONICET. Profesora de la licenciatura el Relaciones Internacionales de la Universidad Nacional de Rosario. Email: julieta.zelicovich@fcpolit.unr.edu.ar
} 
El presente trabajo tiene como meta contribuir al debate acerca de las características, alcances y límites de la Economía Política Internacional (EPI) como perspectiva analítica ${ }^{3}$. En este sentido, se trata de un artículo que está en diálogo con dos trabajos previos publicados en la revista Relaciones Internacionales: el de Tussie (2015), que analiza los orígenes del campo, sus debates y sus líneas rectoras, tanto a nivel general como particular de la Argentina, y el de Peixoto (2017), en el que se argumenta en favor de los puntos de encuentros entre la EPI y el Derecho. En las próximas páginas, se busca resaltar la contribución de la EPI en el área de las Relaciones Internacionales para incrementar la capacidad de cuestionar los procesos de transformación en curso. Para ello, se realiza un recorrido por las preguntas que la EPI permite formular, trascendiendo tanto los análisis de política internacional, como los de economía internacional y los de derecho internacional. Dado que es un campo en formación, y que está en permanente cambio (Blyth, 2009), sostenemos que resulta más lógico centrarse en las preguntas que en las respuestas.

En el primer apartado se define a la EPI, como perspectiva de análisis y campo de investigación, y se explicitan cuáles son los supuestos centrales de la misma. En el segundo apartado, se focaliza en la interrelación entre la EPI y las Relaciones Internacionales y se presenta un conjunto de interrogantes que de ese entrecruzamiento emergen en el contexto de la crisis de globalización, que tiene lugar la segunda década del siglo XXI. Seguidamente, en el tercer apartado, se indaga de qué manera la producción académica latinoamericana ha reflejado este tipo de interrogantes en su agenda de investigación reciente. Para la selección de los artículos reseñados en dicho apartado se hizo un muestreo no aleatorio, focalizado principalmente en artículos publicados en revistas académicas latinoamericanas de ciencias sociales, entre 2015 y 2017, por investigadores radicados en universidades de la región. En algunos casos, se incluyeron también libros o tesis que cumplieran con los mismos requisitos. Cabe indicar que se trata de un recorrido no exhaustivo puesto que el objetivo es ilustrar el avance de nuevas preguntas en el desarrollo disciplinar, antes que realizar un acabo estado de la cuestión de la EPI. Por último, se exponen las conclusiones del artículo.

\section{1. ¿Qué es y qué investiga la Economía Política Internacional?}

La interdependencia entre la economía y la política es lo que distingue a la EPI. ¿Qué significa ello? Que "mientras que los economistas ven a la Economía como un mercado compuesto de fuerzas impersonales, los especialistas en economía política lo interpretan como un sistema sociopolítico integrado por actores con poder" (Gilpin, 2001, p. 38). Es decir, se reconoce que elementos propios de las relaciones de poder y de las instituciones, condicionan el funcionamiento de la economía, y viceversa. Conforme señala Underhill (2000, p.792) tres son las premisas que dan cohesión a la EPI: la primera, que la economía y la política no pueden ser separadas, ni en sentido real, ni con fines analíticos; la segunda, que la interacción política es una de las principales vías a través de la cual las estructuras económicas del mercado se establecen y transforman; y tercero,

\footnotetext{
${ }^{3}$ Agradezco los comentarios de Esteban Actis a este trabajo. Los errores y omisiones que pudieran existir son enteramente de mi autoría
} 
que hay una estrecha conexión entre los niveles de análisis domésticos e internacional. En términos sintéticos, el interrogante que condensa a los estudios de la EPI es: ¿cómo se relacionan las estructuras económicas y la autoridad política (en sentido amplio) en el continuo y acelerado proceso del cambio global? (Underhill, 2000, p 803). Detrás de ello se desagregan otros interrogantes tales como cuáles son los agentes de esa relación, cuáles son los niveles de análisis, cómo se modifica la estructura y cómo se produce la relación entre lo domestico, lo internacional y lo transnacional. Conforme la define Susan Strange, la EPI concierne a los acuerdos sociales, políticos y económicos que afectan al sistema global de producción, intercambio y distribución, y al conjunto de valores que en ello se reflejan; y su estudio, implica analizar las causas profundas, que están enraizadas en cada uno de estos acuerdos (Strange, 1988).

Tal como sostiene Peixoto: "La EPI es un enfoque desde donde mirar al mundo, buscando conexiones invisibilizadas en los enfoques tradicionales de las disciplinas de las que se nutre, proponiendo dejar al descubierto las relaciones de fuerzas, las desigualdades, las voces, los actores, las agendas que contribuyen el orden global, nacional, regional e internacional" (Peixoto, 2017, p. 182). En términos de Seabrooke y Young (2017), la economía política internacional es más bien un campo de investigación, antes que una disciplina. De allí que coexistan diversas perspectivas epistemológicas, ontológicas, teóricas y metodológicas.

Tradicionalmente, se distinguen tres ramas: una liberal, una mercantilista -ligada al realismo- y una estructuralista -próxima a la perspectiva marxista4. Los estudios del pensamiento liberal ponen el foco en una multiplicidad de actores más allá del Estado (y al interior de éste). En efecto, consideran a los agentes individuales, a la vez que asumen que el Estado no es un actor racional unificado sino que en su interior conviven y pujan distintos actores y fuerzas, que se distinguen por sus preferencias o intereses. Sostienen que el sistema internacional es interdependiente, y que las relaciones no necesariamente son de suma cero, sino que de la cooperación pueden obtenerse beneficios mutuos. Desde este enfoque teórico se pregunta por el impacto de las transformaciones sistémicas al interior del Estado, o bien por cómo se realizan los procesos de toma de decisión al interior de los mismos. El enfoque realista considera que el Estado es el actor principal en la EPI y que los actores se rigen por acciones racionales. Considera además, que el sistema internacional es anárquico, y que los Estados se enfrentan en él por poder y riqueza. Las relaciones son, en última instancia, de suma cero. La economía global se subordina a la política internacional. La pregunta central dentro de esta perspectiva es cómo se distribuye el poder entre los Estados. Por su parte, el enfoque estructuralista, también llamado perspectiva crítica, se concentra en los agregados colectivos por oposición al interés nacional, y pone el foco en la estructura del sistema global. Derivado del marxismo, conserva como principal actor de la EPI a las clases sociales. Tanto el Estado como las empresas son instrumentos y expresiones de las clases sociales. Para este enfoque las relaciones son eminentemente conflictivas, y dicho conflicto emana del sistema de producción. Se enmarcan en este enfoque los estudios de la teoría de la dependencia, cuyo epicentro de desarrollo estuvo en América Latina. La agenda de investigación más recien-

\footnotetext{
${ }^{4}$ Debe señalarse sin embargo que en las agendas de investigación más recientes esta distinción esté tendiendo a ser dejada de lado, a la vez que se coloca más énfasis en la trayectoria de investigación según el anclaje geográfico de la producción del conocimiento (Blyth, 2009; Ravenhill, 2014).
} 
te desde esta perspectiva incluye los estudios sobre movimientos laborales, medio ambiente, feminismo, etc.

El origen de la EPI como campo de investigación suele datarse para 1970 en su componente "atlantista", aunque reconoce antecedentes mucho más tempranos si se toman en cuenta perspectivas "del sur", como la teoría de la dependencia. (Cohen, 2008; Weber, 2015). La locación geográfica de cada núcleo de producción académica ha derivado en un conjunto particular de preguntas y conceptos ordenadores. En particular, "Así como la autonomía es el concepto organizador en la experiencia latinoamericana, en la escuela norteamericana lo son la hegemonía y la construcción de normas o regímenes internacionales de manera de proveer estabilidad al orden económico y asegurar el librecambio como piedra basal del dicho orden" (Tussie, 2015, p. 165).

En la Argentina, el desarrollo de la EPI ha estado ligado al debate de la teoría de la dependencia, primero, y de los proceso de integración, después -particularmente en torno al MERCOSUR-y ha tenido un mayor crecimiento en los últimos años, a partir de la incorporación de investigadores que adoptan esta perspectiva dentro del CONICET (Tussie, 2015). Sin embargo, quienes adoptan la EPI como perspectiva analítica no han adquirido aún una identidad propia, sea por reconocimiento propio o por distinción desde otras disciplinas. Una de las razones que llevan a ello es que si bien la EPI tiene un carácter interdisciplinar, nutriéndose tanto de la economía, como del derecho, la política y la sociología; los puntos de encuentro entre los investigadores de estas disciplinas es más bien bajo. Existen así además de los "encapsulamientos" regionales, otros de carácter disciplinar: la EPI de los economistas, la EPI de los internacionalistas, la EPI de los sociólogos, y la EPI de los abogados, por ponerlo de alguna manera. Por ejemplo, si se revisan los programas de los congresos y jornadas académicas de ciencias sociales en la Argentina la EPI es abordada en no más de uno o dos paneles dentro de una gran variedad de cuestiones de las disciplinas de origen. Con la excepción del congreso de Economía Política Internacional, cuya primera edición tuvo lugar en 2014, no se encuentran entrecruzamientos entre diversas formaciones de base en el debate de la EPI, e incluso en ese congreso hay una clara preeminencia de economistas5. De tal forma ese intercambio está aún ausente, y la producción académica se dispersa. La problemática que ello genera es presentada por Blyth del siguiente modo:

After all, one consequence of not being able to 'bound' IPE is that one is bound to run into other academics who are doing essentially the same thing, but not calling what they do IPE. So the obvious question becomes to what extent is this 'the same wine in a different bottle' or a wholly different way of 'doing' IPE? (Blyth, 2009, p.16)

Entrecruzamiento de la EPI y las Relaciones Internacionales frente a la crisis de la globalización

Al centrarnos en el entrecruzamiento de la EPI y las Relaciones Internacionales, debe resaltarse que el aporte de la EPI a la disciplina se ha ido incrementando y ha estado focalizado en la diversidad de interrogantes. Acorde a Tussie y Riggirozi (2015):

Mainstream IPE makes a major contribution to scholarly enquiry by opening up the traditional concerns of IR: the interaction of states; the

\footnotetext{
${ }^{5}$ En la ll edición del Congreso de Economía Política Internacional, del año 2016, sobre un muestreo de las ponencias seleccionadas se observa una presencia prioritaria de economistas (65\%), por sobre otras disciplinas (ciencia política, 5\%; sociología, 5\%, Historia, 10\%; ingeniería, 5\%; no identificado, 10\%).
} 
emergence and operation of multilateral institutions; order and stability in the international system, and the position of the United States as a hegemonic power. This differs from traditional IR in that it introduces a concern with economic factors and market rules in a non-Marxist epistemology, and the belief that these are as important, if not more so, than political and military relations (Tussie, Riggirozi, 2015, p.1045)

Así la EPI contribuye a "correr un velo de ingenuidad" de las RRII respecto al mercado y a cuestionar nociones statusquistas, como la del orden internacional, a la vez que obliga a poner el foco en las fuerzas transnacionales.

En el contexto de transformaciones globales que se vienen sucediendo en la segunda década del siglo XXI, esta perspectiva resulta por demás de relevante, especialmente para los análisis y policy makers "del sur". Y es que en un momento histórico en el que desde los países desarrollados se discuten y ponen en disputa los modos en los que se ordena el sistema internacional, una perspectiva que permita cuestionar las raíces de aquello -material e ideológico- que se produce, se vuelve un imperativo para la formulación de políticas en los países en desarrollo y su comprensión desde la academia, que es interpelada en tal situación ${ }^{6}$.

En efecto, la serie de sucesos que tuvieron lugar desde 2015, constituyen un contexto fértil para el crecimiento y desarrollo de la IPE en la región. Sucesos tales como el Brexit o la elección de Donald Trump en EEUU, fueron la expresión de una serie de transformaciones en curso, tanto de mediano como de largo plazo, que han llevado a plantear la existencia de una "Crisis de la Globalización" (Hale, Held y Young, 2013; Feenstra y Tay-lor, 2013; Sanahuja, 2016; Hass, 2017). Ésta refiere al agotamiento/ralentización/mutación de la globalización -entendida en términos de intensificación de vínculos de comercio y producción que se dio a partir de los '90 con la reducción de las barreras al comercio y la revolución de las tecnologías de la información y comunicación (TICS)-, así como a la ruptura del consenso en torno al internacionalismo liberal, en el que tal expansión de la globalización tuvo lugar. Se trata de un proceso de transformación de las formas de producción, de las dinámicas del comercio, de los polos y fundamentos del poder, de las normas y valores que lo orientan y regulan. Ni los análisis que abordan meramente a las relaciones de poder, ni aquellos que se focalizan en las relaciones económicas por sí solos alcanzan para explicar la realidad en curso. En este marco, la EPI, con su carácter interdisciplinar, cobra relevancia, especialmente para los países del sur que deben encontrar un lugar en ese entorno.

Uno de los interrogantes que se plantea, y en el que desde la EPI pueden producirse reflexiones relevantes, es aquel referido a la gobernanza económica global. Este concepto, se define siguiendo a Keohane y Nye (2000), como el proceso y las instituciones, formales e informales, que orientan y restringen las actividades colectivas de un grupo; remite tanto a la sanción de leyes y normas, como también a la interacción de las fuerzas del mercado y de las instituciones (architecture). En cierto modo es asimilable a la idea de regímenes internacionales. Incluye tanto a actores Estatales, como no estatales, y apunta a las lógicas de la

\footnotetext{
${ }^{6}$ Sobre la vinculación entre la academia y la política, especialmente en temas económicos, véase Botto (2007).
} 
cooperación internacional ${ }^{7}$ (Patrick, 2014). La crisis de globalización implica una crisis de la gobernanza económica global, en tanto altera la forma en la que se producen y ordenan tanto las actividades, como los valores, los actores y las formas de interacción de la economía internacional.

En este marco fenómenos tales como la estructuración y reestructuración de las cadenas globales de valor son analizados dentro de los estudios de la EPI del mainstream "atlántico", así como las negociaciones de los acuerdos de libre comercio en su formato del "regionalismo del siglo XXI" (Baldwin, 2011) y en la modalidad de las negociaciones mega regionales y acuerdos plurilaterales, tanto así como las nuevas clausulas en los tratados bilaterales de inversión (TBI). El rol de las instituciones existentes (negociaciones Ronda Doha en OMC, por ejemplo) entra en tensión con estos nuevos patrones de producción y de regulación, dando lugar a un conjunto de análisis también en crecimiento. Por su parte, se enfoca desde la EPI las propias políticas económicas internacionales de los Estados, las cuales se han ido redefiniendo en el proceso señalado a medida que partidos políticos de cohorte nacionalista y/o proteccionistas llegan al poder, mientras que en otros países, anteriormente menos involucrados en las dinámicas de la gobernanza global, ahora se intensifica su participación en el mundo, como China. En el caso regional, tanto el fin del giro a la izquierda en América Latina, como la crisis brasilera, han sido estudiados en el entrecruzamiento de política internacional y economía internacional.

En estos procesos la EPI tiene gran capacidad para generar preguntas. Tomando como punto de partida los supuestos propios de tal campo de estudio ensayamos algunos planteos: ¿quién detenta el poder en la reestructuración de la gobernanza económica global? ¿Ese poder yace en el Estado, o en el mercado? En todo caso, ¿en qué Estados, respecto de qué sectores del mercado? ¿En qué normas, instituciones, y dinámicas se plasma el proceso de transformación? ¿Quién hace esas normas? ¿Son normas que se enmarcan dentro de instituciones existentes o nuevas instituciones? ¿Qué efectos distributivos generan? ¿Quién las promueve? ¿Por qué quienes acuerdan nuevas modalidades de cooperación o regulación acceden a ello?

Recuperando la tradición latinoamericana de la EPI, con anclaje en la perspectiva de los países del sur, cabe formular un segundo conjunto de preguntas más específicas: ¿cuál es el lugar de los países latinoamericanos en ese proceso? ¿De qué manera esta nueva letra de acuerdos contribuye a la autonomía, la inserción, y el desarrollo de los países de la región? ¿Cómo se relacionan los mismos con las estructuras productivas globales? ¿De qué manera interactúan las empresas transnacionales en esta crisis de la globalización? ¿Y la sociedad civil? ¿Cómo se refleja ello en las dinámicas de integración regional? ¿Quién o quiénes se benefician de este proceso y quiénes se perjudican?

\section{La producción científico-académica latinoamericana de la EPI en el contexto de la crisis de globalización}

Esta serie de preguntas, específicas de la EPI, va permeando paulatinamente la

\footnotetext{
${ }^{7}$ La gobernanza global remite al "esfuerzo colectivo de los Estados soberanos, las organizaciones internacionales y otros actores no estatales para afrontar a los desafíos comunes y aprovechar las oportunidades que trascienden las fronteras nacionales" (Patrick, 2014).
} 
agenda de investigación y producción académica en la región. El número 44 de la revista Pensamiento Propio (julio-diciembre, 2016), del CRIES, es una de las obras que permiten pensar este conjunto de preguntas desde Latinoamérica, con la perspectiva de la EPI. Las revistas académicas de la disciplina, tales como Problemas del Desarrollo y Relaciones Internacionales (ambas de la Universidad Nacional Autónoma de México), Estudios Internacionales (del Instituto de Estudios Internacionales de la Universidad de Chile), Relaciones Internacionales (del Instituto de Relaciones Internacionales de la Universidad Nacional de La Plata, Argentina), Estudos Internacionais (de la Pontifícia Universidade Católica de Minas Gerais, Brasil), también han publicado durante los últimos dos años diversos artículos en los que se abordan partes de estos interrogantes ${ }^{8}$, demostrando una ampliación de las temáticas abordadas en la región desde la EPI, ya no sólo centrada en las cuestiones del desarrollo y la integración regional, sino con miradas sobre una multiplicidad de fenómenos que se derivan del proceso de transformación-crisis en curso.

En las investigaciones relevadas priman los estudios de base empírica, que aluden a las problemáticas de la coyuntura, y sus antecedentes estructurales. Se presentan distintas perspectivas teóricas y metodológicas, las cuales convergen en una serie de regularidades temáticas, según se resume a continuación.

En primer término, sobresale la problematización de la transformación en los polos de poder en la economía internacional y su impacto político. En esa línea, Aronskind (2017) toma los supuestos de la EPI para ahondar en las causas de la crisis de la globalización y lo que él observa como tendencias contracícilicas y su impacto en la región. Su trabajo concluye, por ejemplo, preguntándose "¿a quién representa Trump en el mundo del gran capital? ¿Es el representante de una fracción emergente con poder para sostener una disputa frente al sector "globalizador" de las corporaciones mundiales? ¿O es un fenómeno que refleja una dimensión fuertemente política, habitualmente soslayada por los ideólogos de la globalización, dónde la búsqueda de congruencia económica es secundaria?" (Aronskind, 2017, p.77). Por su parte Mendez-Coto (2016), poniendo el foco en las condiciones previas de la transformación global, se pregunta "¿En qué medida la ampliación del G7 al G20 en la atención de la crisis económica implica un reconocimiento de los cambios en la estructura económica del siglo XXI, particularmente en la influencia de los BRICS en la definición de la gobernanza económica mundial?"

Tomando en cuenta particularmente la presencia de China en la región, desde la EPI se presentan preguntas tales como si ¿existe una transición de una hegemonía norteamericana a una china? Y ¿si el vínculo con China puede ser considerado estratégico para la inserción de los países de América Latina? Y en todo caso, ¿si es autonomizante o dependiente? (Svampa y Slipak, 2015). Bernal-Meza (2016) también indaga en cómo el ascenso de China al rango de potencia económica mundial afectó el desarrollo y la inserción internacional de América Latina. Entrando en un mayor grado de especificidad, y teniendo en cuenta esta creciente vinculación de América Latina con China, en Zelicovich (2017) se pregunta acerca de si los acuerdos de libre comercio firmados por China durante los primeros cinco lustros del siglo XXI han presentado una situación de acoplamientoadhesión al orden vigente o de reforma-transformación de la gobernanza global del comercio internacional. Por su parte, en Paikin y Dulcich (2017) se toma en consideración

\footnotetext{
${ }^{8}$ Trabajos más sistemáticos acerca del estado del campo de la EPI en las publicaciones científicas a nivel global pueden consultarse por ejemplo en Seabrooke and Young (2017)
} 
el impacto del ascenso de China en la región, a partir del análisis de la incidencia de China en las dinámicas de comercio del MERCOSUR.

Este proceso de integración, el MERCOSUR, así como la Alianza del Pacífico (y en su conjunto, la literatura sobre regionalismos) continúan presentes en las reflexiones y producciones de la EPI. Se postulan análisis tanto sobre sobre las cadenas de valor y la integración productiva, como respecto de las cuestiones político-estratégicas de estos procesos, ahora enmarcadas en un contexto de transformaciones globales. Botto, por ejemplo, en un trabajo reciente, recurre a la interacción de fuerzas exógenas y endógenas para explicar las crisis del MERCOSUR, en el contexto de crisis internacional, sosteniendo "la necesidad de superar las explicaciones de índole endógeno (propia de los países miembros o región) para incluir factores exógenos que - como las crisis globales ${ }^{9}-$ intervienen también en los vaivenes de ese proceso" (Botto, 2017, p. 156). Paikin y Perrota (2016) analizan en profundidad las interacciones entre MERCOSUR y la Alianza del Pacífico, recuperando la cuestión del qui bono: se preguntan: “¿en que se beneficiaría concretamente la Argentina con su incorporación a dicha Alianza [del Pacifico]?".

Otro trabajo a resaltar, es el de Vivares y Dolcetti-Marcolini (2015) el cual pone el valor a la perspectiva de la EPI crítica, para considerar las heterogeneidades de los procesos de integración en la región. Si bien no es una investigación en la cual la perspectiva de crisis de globalización esté presente en la pregunta de investigación, sí es valioso el aporte conceptual que realiza como fortalecimiento de la EPI de la región en tal contexto. En la misma línea, también Deciancio (2016) sistematiza los aportes teóricos de la EPI a la literatura del regionalismo, con base en América Latina.

La mirada sobre agentes no estatales es menos frecuente en la EPI que viene produciéndose en la región, pero no inexistente. Halperin, en un trabajo reciente, analiza el papel de las empresas transnacionales en el escenario latinoamericano, poniendo el foco en la relación de esas empresas y sus Estados originarios, y los modos de vinculación entre sector público y el sector privado en función de la inserción de las economías latinoamericanas dentro del sistema global (Halperin, 2017). Giacalone, por su parte, impulsa desde hace tiempo una agenda de investigación con foco en el sector empresario y la EPI. En un trabajo de 2015, se interroga acerca de cómo fue la participación de los sectores empresarios en las negociaciones comerciales, con una perspectiva diacrónica: el G-3 por un lado, y la Alianza del Pacífico por otro; y haciendo un reconocimiento explícito sobre uno de los pilares de la EPI, cual es la interacción de los planos doméstico y sistémico, a la vez que de la relación Estado-Mercado. Desde una lectura de la economía política internacional del marxismo, Delgado Wise y Martin (2015) se preguntan sobre el papel de las diferenciales salariales en la estrategia de reestructuración productiva, comercial, financiera y de servicios impulsada por las grandes corporaciones multinacionales bajo la égida de la globalización neoliberal. Con la excepción del trabajo de Halperin, son muy pocos los trabajos que ponen el foco en la empresa y están atravesados por el contexto de transformación global actual, siendo ésta un área de investigación dentro de la agenda de la EPI en la que se detecta una laguna en la producción científica. La agenda

\footnotetext{
9 "Entre ellas podemos incluir cambios en la regulación global del comercio multilateral, cambios en la direccionalidad de los flujos financieros globales y en la estrategia de inversiones de las empresas transnacionales. Esos cambios de orden global y exógeno generaron fuertes cimbronazos/crisis en el bloque regional que fueron seguidas por procesos de deconstrucción institucional" (Botto, 2017:172)
} 
se amplía si se focalizan otras temáticas, como la de los medios de comunicación (Martens, Vivares y McChesney, 2014; Schleifer, 2013), o el delito (Pontón Cevallos, 2016).

Por su parte, cobran relevancia estudios que ponen el foco en la relación EstadoMercado, por ejemplo, en la relación entre Estado, empresas transnacionales y su marco jurídico y político. Arroyo Picard y Ghiotto (2017) se preguntan: “¿por qué Brasil, que durante alrededor de 25 años se negó a ratificar sus tratados sobre inversiones, ahora avanza en la firma de nuevos tratados?". Por su parte, Actis (2016) analiza el vínculo entre Brasil y las empresas multinacionales desde una lectura político-estratégica, reflexionando en torno a si "¿¿l accionar externo del Estado brasileño fue un elemento que permitió consolidar y potenciar el proceso de internacionalización brasileño entre los años 2003 y 2010? O por el contrario, ¿las inversiones de las compañías brasileñas en terceros mercados fueron funcionales a una estrategia particular de la PEB?" 10

Los regímenes internacionales y tratados internacionales en cuestiones financieras y de comercio constituyen también una agenda fructífera para el entrecruzamiento de EPI y Relaciones Internacionales en el contexto actual en la región. Se trata de uno de los nuevos más tradicionales de la EPI, que es replicado, redefinido y ampliado con una perspectiva propia de los países en desarrollo. Por ejemplo, Nemiña (2017) cuestiona las dinámicas de negociaciones entre FMI y países en desarrollo, señalando que "Si bien EE.UU. posee una posición dominante dentro del organismo, su acción suele tomar en cuenta la posición de las demás potencias y la burocracia del FMI. Asimismo, la respuesta de los gobiernos frente a un acuerdo refleja el equilibrio entre los intereses de la administración y los actores políticos internos." (Nemiña, 2017, p.22). Así, concluye, "la interrelación entre estructura y agencia, la crítica al actor racional y la consideración de los intereses como condicionantes de la acción permiten considerar la relación entre el FMI y los países en desarrollo como un vínculo complejo y dinámico, cuyo resultado no está determinado de antemano." (Nemiña, 2017, p.22).

Una problemática específica de Latinoamérica y que tiene lugar en el contexto de antesala de la crisis de globalización es la del endeudamiento y la reforma de la arquitectura financiera. Salgado Rodrigues (2016) plantea "¿cuáles son las bases de una nueva arquitectura financiera? En ello, aludiendo al contexto de transformación global ¿Cuál es el papel de los países BRIC en el mundo y del Banco del Sur en el contexto latinoamericano del siglo XXI?" En torno a los procesos de endeudamiento existen miradas sobre su relación con el desarrollo: "¿por qué, con pocas excepciones, las deudas acumuladas por los países en desarrollo no pudieron ser amortizadas en la segunda parte del siglo XX?" (Mango y Aschieri, 2015); “¿cuál es la diferencia entre endeudamiento de los países pobres con el endeudamiento de los países ricos?", poniendo el foco en el "poder de deuda" y "poder de deudor" (Aschieri y Jedlicki, 2014); “¿cuál es la relación entre el crédito externo (en divisas) y las posibilidades de contribuir al desarrollo capitalista en un país periférico como la Argentina?" (Gosparini, Sanchez y Tavilla, 2014). Por su parte, Fernández Alonso y Stanley (2017)

\footnotetext{
${ }^{10}$ Existen otros trabajos, publicados ya no en revistas académicas sino como tesis de grado y posgrado, que exploran más casos del accionar de empresas multinacionales en relación a la política exterior del Estado. Tal es el caso de Aldana Chillo (2015) "Petrobras y PDVSA en Ecuador (2008-2010): softpower y poder estructural de las empresas multinacionales" y Cortés Saenz (2015) "El petróleo como recurso de poder e instrumento de política exterior a partir de la noción de poder estructural de Susan Strange. Venezuela en Post Guerra Fría"
} 
analizan los retos que enfrentan los países sudamericanos para acceder a la financiación del desarrollo, particularmente ¿cuáles son las condicionalidades que los países sudamericanos tienen para afrontar el acceso a dicho financiamiento?

Las negociaciones comerciales multilaterales, y la participación de los países en desarrollo dentro de las mismas, vienen siendo analizadas fundamentalmente por Tussie en sucesivos trabajos desde la década del 1980: “¿Cómo responden y se ajustan los países menos desarrollados al orden comercial reflejado en el GATT?" (Tussie, 1993); "How can developing countries manage the terms of integration into the global economy? How do different sets of external pressures place constraints on national development strategies? What offsetting mechanisms have emerged?" (Saguier y Tussie, 2014). En el contexto actual de transformaciones y crisis de globalización, se han retomado tales interrogantes y se han realizado esfuerzos por comprender el avance de las negociaciones de la Organización Multilateral de Comercio desde la perspectiva de la EPI. Los interrogantes son “¿Cómo se pasó del estancamiento de la Ronda Doha a una instancia más dinámica de las negociaciones durante las Conferencias Ministeriales de Bali y Nairobi? ¿Se trata de un cambio estructural o de resultados coyunturales?" (Zelicovich, 2017) “¿cuáles son las implicancias del acuerdo de Nairobi para la debatida gobernanza global del comercio internacional?" (Zelicovich, 2016). La tradición de investigación desde EPI en relación a los países en desarrollo dentro de estos organismos es abordada también en función de las transformaciones reciente, así se plantea, ¿cómo pueden lograr países, como los BRICS, incidir en la reforma de la OMC y la gobernanza global? (Delich, 2016).

Este breve ejercicio de recorrida por la EPI de la región-que dista claramente de ser un relevamiento exhaustivo del estado del arte- basta para mostrar el crecimiento de su campo de investigación y sus aportes para las relaciones internacionales. Al tomar a la EPI como perspectiva de análisis del proceso de "crisis de la globalización" en curso es posible incrementar la lectura crítica, y aprender los cursos más profundos de transformación, y su impacto regional. La interacción entre economía y política permite ahondar en una compresión del proceso de reacomodo del orden internacional, que no se queda ni en el diseño institucional, ni en los documentos firmados, ni en la evolución de flujos de comercio, IED y PBI, sino en el entrecruzamiento de todos estos aspectos. La perspectiva también permite formular recomendaciones de políticas que permitan a los países del sur global tener más opciones q una adscripción y aceptación del orden internacional en formación.

\section{Reflexiones finales}

La EPI tiene una gran capacidad para formular preguntas, y es a partir de éstas que el conocimiento avanza. Si se toma la experiencia de otros campos, como la filosofía política, es notorio el potencial que la EPI tiene para el desarrollo de los estudios de las Relaciones Internacionales.

Al ver las experiencias políticas comunes desde un ángulo ligeramente diferente al prevaleciente, al formular una antigua pregunta en una forma novedosa, al rebelarse contra las tendencias conservadoras del pensamiento y el lenguaje, los pensadores han contribuido a liberar las formas establecidas de pensamiento y a imponer a sus con- 
temporáneos y a la posteridad la necesidad de repensar la experiencia política (Wolin, 2012, p. 46)

En este trabajo hemos intentando recuperar cuál es el conjunto de preguntas que desde la EPI se plantea para comprender la transformación global contemporánea. Queda de manifiesto que la misma ha servido como incentivo para ampliar la agenda de investigación en América Latina, y en consecuencia ir fortaleciendo el desarrollo de este campo de investigaciones en las Relaciones Internacionales. Existen, no obstante, numerosas lagunas - preguntas sin responder- y una agenda institucional por ser desarrollada, a fin de generar puntos de entrecruzamiento y reconocimiento de las distintas disciplinas que nutren la EPI de la región, en general, y de Argentina, en particular.

\section{Referencias bibliográficas}

- Actis, Esteban (2016), “¿Qué fue primero, el huevo o la gallina? Política exterior e internacionalización de capitales en Brasil bajo los gobiernos de "Lula" da Silva", Revista POSTData: Revista de Reflexión y Análisis Político, vol. 21, núm. 2, pp. 393-421.

- Aldana Chillo (2015), “Petrobras y PDVSA en Ecuador (2008-2010): softpower y poder estructural de las empresas multinacionales" Monografía presentada como exigencia para obtención de grado de Bacharelado en Relaciones Internacionales e Integración de la Universidad Federal De Integración Latinoamericana. Disponible en https://dspace.unila.edu.br/handle/123456789/483

- Aronskind, Ricardo (2017), "Trump: ¿un parche nacionalista a la crisis de la globalización?" Revista Estado y Políticas Públicas, no 8, pp. 59-79

- Arroyo Picard, Alberto y Ghiotto, Luciana (2017) "Brasil y la nueva generación de Acuerdos de cooperación y facilitación de inversiones: un análisis del Tratado con México" en Relaciones Internacionales, $n^{\circ} 52$, pp 39-54.

- Aschieri, Enrique y Jedlicki, Claudio (2014) “Poder de la deuda y poder del deudor. La hegemonía del dólar revisitada" Ponencia presentada en el I Congreso de Economía Política Internacional. Disponible en http://www.unm.edu.ar/congresoeconomiainternacional/ponencias/aschieri.pdf

- Baldwin, Richard (2011), "21st century regionalism: Filling the gap between 21st century trade and 20th century trade rules", World Trade Organization Economic Research and Statistics Division, ${ }^{\circ}$ ERSD-2011-08.

- Bernal-Meza, Raúl (2016), “China y América Latina: de la oportunidad al desafío”, Revista tempo do mundo, $v 2, n^{\circ} 2, p p$ 63-78.

- Blyth, Mark (2009), Routledge Handbook of International Political Economy. New York: Routledge.

- Botto, Mercedes (2007), Saber y política en América Latina. El uso del conocimiento en las negociaciones comerciales internacionales. Buenos Aires: Prometeo Libros

- Botto, Mercedes (2017). “El Mercosur y sus crisis: análisis de interpretaciones sobre el fracaso de la integración regional sudamericana", Estado \& Comunes, revista de políticas y problemas públicos, $n^{\circ} 5$, vol 2. Pp 155-176. 
- Cohen, Benjamin (2008), International Political Economy: an intellectual history. Princeton: Princeton University Press.

- Cortés Saenz (2015), “El petróleo como recurso de poder e instrumento de política exterior a partir de la noción de poder estructural de Susan Strange. Venezuela en Post Guerra Fría" Universitat Autònoma de Barcelona. Departament de Dret Públic i de Ciències Historicojurídiques. Disponible en http://hdl.handle.net/10803/382480

- Deciancio, Melisa (2016), “El regionalismo latinoamericano en la agenda de la teoría de las Relaciones Internacionales" Iberoamericana, vol 16, $n^{\circ} 63$, pp 91-110

- Delgado Wise, Raúl y Martin, David (2015), "La Economía política del arbitraje laboral global”, Revista problemas del desarrollo, $n^{\circ} 183$, vol 46. Pp 13-32

- Delich, Valentina (2016), “Gobernanza Global y BRICS: reformando la OMC a fuego lento" en Pelfini, Alejandro y Fulquet, Gastón (coord.), Los BRICS en la construcción de la multipolaridad. ¿'Reforma o adaptación?. Buenos Aires: CLACSO. Pp. 51-62

- Feenstra, Robert; Taylor; Alan (2013). Globalization in an Age of Crisis: Multilateral Economic Cooperation in the Twenty-First Century. Chicago: University of Chicago Press

- Fernandez Alonso, José y Stanley, Leonardo (2017), "The changing problems of regional development finance in Latin America" mimeo

- Giacalone, Rita (2015), “Comparación de la participación empresarial colombiana en las negociaciones del G-3 y la Alianza del Pacífico", Revista Aportes para la Integración Latinoamericana, vol 11, n³2. Pp 1-28.

- Gilpin, Robert (2001), Global Political Economy. Understanding the International Economic Order. Princeton: Princeton University Press, 2001.

- Gosparini, Florencia; Sanchez, Esteban y Tavilla, Pablo (2014). “Hegemonía financiera internacional, regímenes de acumulación y endeudamiento externo en la periferia". Ponencia presentada en el I Congreso de Economía Política Internacional. Disponible en http://www.unm.edu.ar/congresoeconomiainternacional/ponencias/gosparini.pdf

- Hale, Thomas; Held, David; Young, Kevin (2013). Gridlock: Why Global Cooperation Is Failing When We Need It Most. Cambridge: Polity Press.

- Halperin, Marcelo (2017), "Las empresas transnacionales en el escenario latinoamericano del capitalismo tardío", Revista Aportes para la integración Latinoamericana". Vol 23, № 36, pp 1-43.

- Hass, Richard (2017). A world in disarray: American foreign policy and the crisis of the old order. New York: Penguin Press.

- Keohane, Robert y Nye, Joseph (2000) "Introduction" en Dohahue, J. (ed). Governance in a globalizing world. Washington, D.C: Brookings Institution Press.

- Mango, Francisco y Aschieri, Enrique (2015). “El Ciclo-D: déficit, deuda y default en los países en desarrollo en el orden económico internacional actual". Revista Argentina de Economía Internacional, $n^{\circ} 4, \mathrm{pp}$ 3-30. 
- Martens, Cheryl; Vivares, Ernesto y McChesney, Robert (2014), The international political economy of communication. Media and power in South America. New York: Palgrave.

- Mendez-Coto, Marco Vinicio (2016). “Multilateralismo, gobernanza y hegemonía en la estructura económica internacional: del G7 al G20" en Relaciones Internacionales, GERI, $n^{\circ} 31$. Pp 13-32

- Nemiña, Pablo; (2017), “Aportes de la Economía Política internacional a la comprensión teórica de la relación entre el FMI y los países en desarrollo", Civitas - Revista de Ciências Sociais, $n^{\circ} 17$, pp. 11-25

- Paikin, Damian y Dulcich, Federico (2017) "El sexto socio del MERCOSUR: Un estudio sobre la penetración importadora de china y su impacto en el comercio intrarregional", Revista Perspectivas de Políticas Públicas, v. 6, n 12, pp 395-414

- Paikin, Damián y Perrota, Daniela (2016). "La Argentina y la Alianza del Pacífico: riesgos y oportunidades de una nueva geopolítica", Revista aportes para la integración latinoamericana, $n^{\circ} 34, \mathrm{pp}$ 67-101.

- Patrick, Stuart (2014) "The Unruled World. The Case for Good Enough Global Governance", Foreign Affairs, January/February 2014. Disponible en: https://www.foreignaffairs.com/articles/2013-12-06/unruled-world

- Peixoto Batista, Juliana. (2017), “La EPI y las Relaciones Internacionales, ¿Dónde está el Derecho?". Relaciones Internacionales, v. 26, n², pp 181-194

- Pontón Cevallos, Daniel (2016). “Perspectivas y dilemas para una compresión económica del delito organizado transnacional”, Estado \& comunes. Revista de políticas y problemas públicos. n. ${ }^{\circ}$, pp. 115-134.

- Ravenhill, John (2014), Global Political Economy. Oxford: Oxford University Press

- Saguier, Marcelo y Tussie, Diana (2014) “Emerging trade politics: the continuos pendulum from multilateralism to asymmetric trade negotiations", Revista estudos internacionais, v. $2, n^{\circ} 1$, pp. 9-26

- Salgado Rodrigues, Bernardo (2016). "La nueva arquitectura financiera mundial: perspectivas del Banco de los BRICS y del Banco del Sur". Ponencia presentada en el II Congreso de Economía Política Internacional. Disponible en https://goo.gl/mVUCyE

- Sanahuja, José Antonio (2016), “América Latina en un cambio de escenario: de la bonanza de las commodities a la crisis de la globalización", Revista Pensamiento Propio, v $21, n^{\circ} 44$.

- Seabrooke, Leonard y Young, Kevin (2017), "The networks and niches of international political economy", Review of International Political Economy, v. 24, n², pp. 288-331.

- Strange, Susan (1988) State and Markets. London: Pinter

- Svampa, Maristella y Slipak, Ariel (2015). “China en América Latina: Del Consenso de los Commodities al Consenso de Beijing", Revista Ensambles primavera 2015, año 2, n.3, pp. 34-63 
- Tussie, Diana (1993), Los países menos desarrollados y el sistema de comercio mundial, México, D.F.: Fondo de Cultura Económica.

- Tussie, Diana (2015) “Relaciones Internacionales y Economía Política Internacional: notas para el debate" Relaciones Internacionales, $n^{\circ} 48$, pp 155-175

- Tussie, Diana y Riggirozi, Pía (2015). “A global conversation: rethinking IPE in post-hegemonic scenarios" Contexto Internacional, v. 37, n³, pp. 1041-1068

- Underhill, Geoffrey (2000). "State, market and global political economy: genealogy of an (inter-?) discipline" International Affairs, $n^{\circ} 76$, v 4, pp 791-810

-Vivares, Ernesto y Dolcetti-Marcolini, Michele (2016) "Two regionalisms, two Latin Americas or beyond Latin America? Contributions from a critical and decolonial IPE", Third World Quarterly, n³7, v 5, pp 866-882,

- Weber, Heloise (2015). “Is IPE just 'boring', or committed to problematic meta-theoretical assumptions? A critical engagement with the politics of method", Contexto Internacional, v.. 37, $\mathrm{n}^{\circ} 3$, pp. 913-944.

- Wolin, Sheldon (2012) Política y Perspectiva. Continuidad e innovación en el pensamiento político occidental. México: Fondo de Cultura Económica

- Zelicovich, Julieta (2016) “La economía política internacional en las negociaciones multilaterales de agricultura. El caso de las subvenciones a las exportaciones agrícolas en la Ronda Doha." en Revista de Estudios Sociales Contemporáneos no 14, IMESC-IDEHESI/Conicet, Universidad Nacional De Cuyo, 2016, pp. 64-84

- Zelicovich, Julieta (2017). “¿Esta viva la OMC? Bloqueos y consensos en las negociaciones de las Conferencias Ministeriales de Bali y Nairobi”. UNISCI Journal, $n^{\circ} 44$, pp 137162

-Zelicovich, Julieta (2017). “China, los tratados de libre comercio y la gobernanza global del comercio internacional en el siglo XXI", Estudos internacionais, v.4 n.3, pp.113 129 